\title{
Serum anti-proteinase 3 anti-neutrophil cytoplasmic antibody in ulcerative colitis: a potential marker of disease activity that predicts steroid therapy failure
}

\section{Yuki Aoyama}

Kagawa Prefectural Central Hospital

Tomoki Inaba

Kagawa Prefectural Central Hospital

Sakuma Takahashi ( $\nabla$ saqi@yahoo.co.jp )

Kagawa Prefectural Central Hospital https://orcid.org/0000-0001-8533-3549

Hisae Yasuhara

Mitoyo General Hospital

\section{Sakiko Hiraoka}

Okayama University Graduate School of medicine, Dentisty and Pharmaceutical Sciences

Koichi Izumikawa

Kagawa Prefectural Central Hospital

Shigenao Ishikawa

Kagawa Prefectural Central Hospital

\section{Masaki Wato}

Kagawa Prefectural Central Hospital

Midori Ando

Kagawa Prefectural Central Hospital

Satoko Nakamura

Kagawa Prefectural Central Hospital

Koichi Mizobuchi

Kagawa Prefectural Central Hospital

Hiroyuki Okada

Okayama University Graduate School of medicine, Dentistry and Pharmaceutical Sciences

Research article

Keywords: ulcerative colitis, PR3-ANCA, disease activity, steroid therapy, colonoscopy

Posted Date: August 6th, 2020

DOl: https://doi.org/10.21203/rs.3.rs-49488/v1 
License: (c) (i) This work is licensed under a Creative Commons Attribution 4.0 International License. Read Full License 


\section{Abstract}

Background Serum anti-proteinase 3 anti-neutrophil cytoplasmic antibody (PR3-ANCA) is a diseasespecific antibody against granulomatosis with polyangiitis. Some patients with ulcerative colitis (UC) have positive results for PR3-ANCA but the clinical significance of PR3-ANCA is not clear. Thus, we conducted a multicenter, retrospective, observational study to elucidate the clinical significance of PR3ANCA in UC.

Methods In total, 150 patients with UC underwent colonoscopy and serum PR3-ANCA measurements. Activity was evaluated using the Mayo Endoscopic Subscore (MES), and the relationship between activity and PR3-ANCA was analyzed.

Results Twenty-six of the 150 patients who met the exclusion criteria were eliminated; 124 patients were included. A positive correlation was observed between MES and PR3-ANCA $(r=0.42 ; p<0.001)$. Receiveroperating characteristic analysis showed that the cut-off value for calculations was $4.1 \mathrm{U} / \mathrm{mL}$. Of 108 patients with active-phase UC, 58 (53.7\%) were positive for PR3-ANCA. Furthermore, patients for whom steroid therapy was ineffective had a significantly higher rate of being PR3-ANCA positive $(p=0.045)$. Of 21 patients who reached clinical remission with PR3-ANCA-positive active-phase UC, 13 had an MES $\geq 1$ and 8 patients had an MES 0 . For MES 0 cases, the reduction of PR3-ANCA levels was significant $(p=$ 0.012) when comparing the active-phase and clinical remission. All cases with MES 0 were negative for PR3-ANCA. Only 4 of 13 cases with MES $\geq 1$ were negative for PR3-ANCA.

Conclusions Approximately half of UC cases are PR3-ANCA-positive during the acute phase, indicating that PR3-ANCA is a potential marker of disease activity that predicts steroid therapy failure.

Trial registration This study was registered in the UMIN Clinical Trials Registry System (000039174). Retrospectively registered on 16th Jan. 2020.

\section{Introduction}

Ulcerative colitis (UC) is a chronic, refractory inflammatory bowel disease of unknown cause and is diagnosed either histologically or by colonoscopy[1]. Clinical remission has been the primary target of treatment; however, endoscopic remission (when endoscopy shows no mucosal inflammation) has recently become more appropriate[2]. Endoscopic evaluation would be desirable to accurately evaluate disease activity. Nonetheless, the significant physical and financial burdens make it difficult to perform frequent endoscopic examinations. Fecal hemoglobin and calprotectin have recently been reported as useful for evaluating disease activity $[3,4]$. Furthermore, the ability to use blood test results to evaluate disease activity would be extremely beneficial in clinical practice.

Serum ANCA is a general term for anti-neutrophil cytoplasmic antibodies. Clinically important ANCAs include cytoplasmic ANCAs (c-ANCA or PR3-ANCA), which target the antigen proteinase 3 (PR3), and perinuclear ANCAs ( $\mathrm{p}-\mathrm{ANCA}$ or MPO-ANCA), which target the antigen myeloperoxidase (MPO). Anti- 
proteinase 3 (PR3) is a highly specific biomarker for granulomatosis with polyangiitis[5], and MPO-ANCA is a highly specific biomarker for eosinophilic granulomatosis with polyangiitis or microscopic polyangiitis[6]. To diagnose UC, ANCA is measured to exclude intestinal lesions caused by vasculitis. MPO-ANCA is reportedly useful to differentiate UC from Crohn's disease because UC cases in Western countries are often positive for MPO-ANCA[7, 8]. However, in Japan and other regions of Asia, UC cases are commonly positive for PR3-ANCA, not MPO-ANCA[9, 10]. A multicenter, retrospective, observational study was conducted to elucidate the clinical significance of PR3-ANCA in UC.

\section{Materials And Methods}

\section{Patients.}

Between April 2016 and June 2019, Kagawa Prefectural Central Hospital and Mitoyo General Hospital registered 150 patients with UC who underwent colonoscopy and PR3-ANCA measurement within 1 week. Patients were excluded for the following reasons: age 5 years or younger; a complication that could be positive for PR3-ANCA or MPO-ANCA such as autoimmune disease, chronic extraintestinal inflammatory disease[11], or malignant tumor[12]; and undergoing treatment with or having a history of treatment with tumor necrosis factor (TNF)-a inhibitors (infliximab, adalimumab and golimumab) [13], which have the potential to cause drug-induced ANCA-associated vasculitis.

UC was diagnosed based on the Lennard-Janes criteria[14]. Disease types were classified based on the Montreal Classification[15] as proctitis, left-sided, and extensive. Disease activity was evaluated by assigning the Mayo Endoscopic Subscore (MES)[16] to the sites with the most severely inflamed mucosa, according to colonoscopy results. An MES of 0 was defined as remission and an MES $\geq 1$ was defined as active-phase disease. A cavitating ulcer was defined as an ulcer that reached the muscular layer with a margin that looked like it was cut into the shape of a cliff according to endoscopic findings. A cavitating ulcer is one of the hallmarks of severe UC and poses a risk of perforation. Pathological activity was evaluated by assigning the Matts grade[17] to biopsy results of the site with the most severely inflamed mucosa. The MES and Matts grade were evaluated by two blinded individuals (two endoscopy specialists and two pathologists).

Clinical activity was evaluated using the Partial Mayo Score, and a score $\leq 1$ was defined as clinical remission. We examined the response to the therapy for the most severe flare-up of colitis in each participant. Those who achieved clinical remission within 6 months of treatment were considered responders. PR3-ANCA status of responders or non-responders to each therapy was evaluated.

Steroid resistance has been defined as an exhibition of no clinical improvement after treatment with highdose oral steroids (40-60 mg/day prednisone or equivalent) within 30 days or no clinical improvement after treatment with high-dose intravenous prednisone within 7-10 days[18]. Steroid dependence has been defined by an initial response to treat with high-dose prednisone but then relapse during tapering or shortly after drug discontinuation and requiring re-introduction to maintain symptom control[19]. 
PR3-ANCA and MPO-ANCA were measured at an external institution using a kit (STACIA® MEBLux ${ }^{\mathrm{TM}}$ test; $\mathrm{MBL}$ ) including a chemiluminescent enzyme immunoassay (CLEIA) on serum that was cryopreserved at $-10{ }^{\circ} \mathrm{C}$ or below. The detection limit value for PR3-ANCA and MPO-ANCA was $1.0 \mathrm{U} / \mathrm{mL}$. Cytomegalovirus $(\mathrm{CMV})$ infection was defined as positive if either CMV immunostaining of biopsy tissue or serum CMV pp65 antigen (C7-HRP) was positive.

A specialist in collagen diseases confirmed that all patients did not have granulomatosis with polyangiitis during the course of their UC. The primary endpoint was the relationship between UC activity and PR3-ANCA.

The UMIN registration number of this study is 000039174.

\section{Statistical Analysis.}

EZR (version 3.4.1 for Windows) was used for statistical analysis. Fisher's exact test and chi-squared test were used for nominal variables, and Mann-Whitney $U$ test and Student t-test were used for continuous variables. Spearman rank correlation or Pearson correlation were used for correlation analysis. Receiveroperating characteristic (ROC) analysis was conducted to estimate the cut-off value for PR3-ANCA. The level of statistical significance was set to $p<0.05$.

\section{Results}

Of 150 UC patients, 124 were analyzed; 12 were excluded for possessing an autoimmune disease, chronic extraintestinal inflammatory disease, or malignant tumor; 14 for undergoing treatment with a history of treatment with TNF-a inhibitors. Of the 124 patients, 30 (24.2\%) presented with their initial attack of colitis.

Patients included 65 men and 59 women with a mean age of 42.4 years $( \pm 16.9$ years). The mean age of onset was 35.2 years ( \pm 16.0 years), and the median disease duration was 3.5 years $(0.8-12.0)$. There were 85 cases of extensive colitis (68.5\%), 25 cases of left-sided colitis (20.2\%), and 14 cases of proctitis (11.3\%) (Table 1). Sixteen patients had an MES of 0, 30 had an MES of 1, 33 had an MES of 2, and 45 had an MES of 3, indicating 16 cases in remission (12.9\%) and 108 cases in the active-phase (87.1\%). 
Table 1

Characteristics of subjects

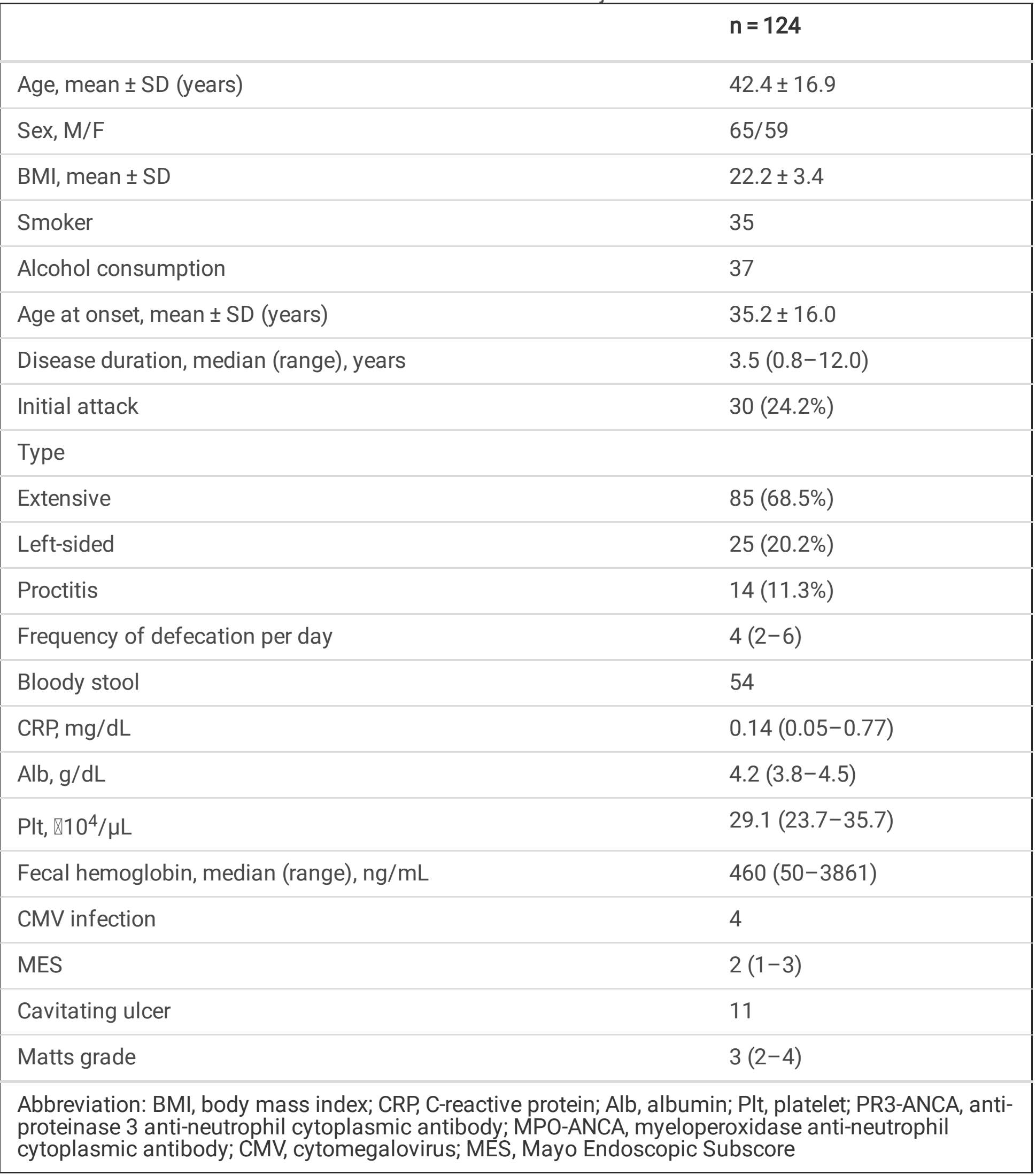

Table 2 shows patients' PR3-ANCA or MPO-ANCA levels grouped by MES. Of the 108 active-phase cases, 77 cases $(71.3 \%)$ had PR3-ANCA $\geq 1.0 \mathrm{U} / \mathrm{mL}$, and 7 (6.5\%) had MPO-ANCA $\geq 1.0 \mathrm{U} / \mathrm{mL}$. When PR3-ANCA 
was $<1.0 \mathrm{U} / \mathrm{mL}$, MPO-ANCA was also $<1.0 \mathrm{U} / \mathrm{mL}$, and all patients with MPO-ANCA $\geq 1.0 \mathrm{U} / \mathrm{mL}$ also had PR3-ANCA $\geq 1.0 \mathrm{U} / \mathrm{mL}$.

Table 2

Patients' PR3-ANCA and MPO-ANCA levels by MES group

\begin{tabular}{|c|c|c|c|c|}
\hline MES & $0(n=16)$ & $1(n=30)$ & $2(n=33)$ & $3(n=45)$ \\
\hline PR3-ANCA $(\geqq 1.0)$ & 6 & 15 & 23 & 39 \\
\hline 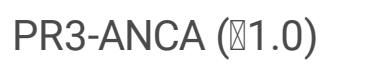 & 10 & 15 & 10 & 6 \\
\hline MPO-ANCA $(\geqq 1.0)$ & 1 & 2 & 3 & 2 \\
\hline MPO-ANCA $(₫ 1.0)$ & 15 & 28 & 30 & 43 \\
\hline
\end{tabular}

A positive correlation was observed between MES and serum PR3-ANCA levels ( $r=0.42 ; 95 \%$ confidence interval [Cl], 0.27-0.56; $\mathrm{p}<0.001$ ) (shown in Fig. 1). ROC analysis showed that the cut-off value was 4.1 $\mathrm{U} / \mathrm{mL}$ (area under the curve [AUC], $0.72 ; 95 \% \mathrm{Cl}, 0.60-0.83$; sensitivity, 0.88 ; specificity, 0.54 ) (shown in Fig. 2).

Of 108 patients with cases in the active-phase, 58 patients (53.7\%) had serum PR3-ANCA $\geq 4.1 \mathrm{U} / \mathrm{mL}$, indicating the PR3-ANCA-positive group, and 50 patients (46.3\%) had serum PR3-ANCA $<4.1 \mathrm{U} / \mathrm{mL}$, indicating the negative group. A comparison of the PR3-ANCA in the active-phase showed that the PR3ANCA-positive group had significantly higher MES, pathological activity, C-reactive protein (CRP), frequency of defecation, and

lower albumin (Alb) ( $p=0.005,0.005,0.017,0.048$, and 0.003 , respectively) than the negative group (Table 3). 
Table 3

Comparison of PR3-ANCA-positive group and negative group in the active phase

\begin{tabular}{|c|c|c|c|}
\hline & $\begin{array}{l}\text { PR3-ANCA } \geqq 4.1 \\
(n=58)\end{array}$ & $\begin{array}{l}\text { PR3-ANCA囚4.1 } \\
(n=50)\end{array}$ & p-value \\
\hline Age, mean \pm SD (years) & $41.3 \pm 17.8$ & $43.0 \pm 17.3$ & 0.610 \\
\hline Sex, M/F & $29 / 29$ & $28 / 22$ & 0.570 \\
\hline $\mathrm{BMI}$, mean $\pm \mathrm{SD}$ & $22.2 \pm 3.2$ & $22.3 \pm 3.8$ & 0.940 \\
\hline Smoker & 13 & 15 & 0.390 \\
\hline Alcohol consumption & 15 & 13 & 1 \\
\hline Age at onset, mean \pm SD (years) & $36.2 \pm 17.1$ & $35.4 \pm 15.9$ & 0.800 \\
\hline Disease duration, median (range), years & $2(0-7)$ & $3.5(0-12.8)$ & 0.280 \\
\hline Type & & & 0.320 \\
\hline Extensive & 45 & 32 & 0.140 \\
\hline Left-sided & 9 & 11 & 0.460 \\
\hline Proctitis & 4 & 7 & 0.340 \\
\hline Frequency of defecation per day & $5(2-10)$ & $4(2-5)$ & 0.048 \\
\hline Bloody stool & 30 & 24 & 0.850 \\
\hline $\mathrm{CRP}, \mathrm{mg} / \mathrm{dL}$ & $0.34(0.09-2.7)$ & $0.12(0.04-0.57)$ & 0.017 \\
\hline Alb, g/dL & $3.9(3.2-4.3)$ & $4.3(4.0-4.6)$ & 0.003 \\
\hline $\mathrm{Plt}, \times 10^{4} / \mu \mathrm{L}$ & $30.0(25.6-40.3)$ & $29.9(23.8-35.0)$ & 0.480 \\
\hline Fecal hemoglobin, median (range), ng/mL & $763(50-4744)$ & $890(136-4256)$ & 0.940 \\
\hline CMV infection & 3 & 1 & 0.620 \\
\hline MES & $3(2-3)$ & $2(1-3)$ & 0.005 \\
\hline Cavitating ulcer & 9 & 2 & 0.140 \\
\hline Matts grade & $4(3-5)$ & $3(2-4)$ & 0.005 \\
\hline
\end{tabular}

Table 4 shows the stratification based on PR3-ANCA status of responders and non-responders to induction therapy, among a total of 108 patients during their most severe flare-up of colitis. Steroid therapy was introduced to 37 cases with a median Partial Mayo Score of 5 (4-7). In non-responders to 
steroid therapy, leukocytapheresis (LCAP) or tacrolimus was used as additional therapy. Median Partial Mayo Scores of responders and non-responders were $5(4-7)$ and 4 (3-6) respectively and were not significantly different between the two groups $(p=0.55)$. Of the 18 patients who failed to achieve clinical remission to steroid therapy (48.6\%), 5 were steroid-resistant and 13 steroid-dependent. PR3-ANCA positivity of non-responders to steroid therapy was significantly higher than that of responders $(77.8 \% \mathrm{vs}$. $42.1 \%, p=0.045)$.

Table 4

Positive rates of PR3-ANCA in responders or non-responders to the past therapy during the most severe flare-up of colitis

\begin{tabular}{|cccc|}
\hline & Responders & Non -responders & p-value \\
\hline & $(\mathrm{n}=68)$ & $(\mathrm{n}=40)$ & \\
\hline Past therapy & & & \\
\hline 5-ASA alone & $24 / 49(49.0 \%)$ & $11 / 21(52.4 \%)$ & 1 \\
\hline LCAP alone & $0 / 0(0.0 \%)$ & $1 / 1(100.0 \%)$ & 1 \\
\hline Steroid & $8 / 19(42.1 \%)$ & $14 / 18(77.8 \%)$ & 0.045 \\
\hline Alone & $3 / 9(33.3 \%)$ & $0 / 2(0.0 \%)$ & 1 \\
\hline Plus, 5-ASA & $5 / 10(50.0 \%)$ & $8 / 9(88.9 \%)$ & 0.14 \\
\hline Plus, 5-ASA plus LCAP & $0 / 0(0.0 \%)$ & $3 / 4(75.0 \%)$ & 1 \\
\hline Plus, tacrolimus & $0 / 0(0.0 \%)$ & $3 / 3(100.0 \%)$ & 1 \\
\hline $\begin{array}{l}\text { Abbreviation: PR3-ANCA, anti-proteinase 3 anti-neutrophil cytoplasmic antibody; 5-ASA, 5- } \\
\text { Aminosalicylic Acid; LCAP, leukocytapheresis }\end{array}$ & \\
\hline
\end{tabular}

Of the 124 cases, 16 had a Matts grade of 1 (12.9\%), 20 had a Matts grade of 2 (16.1\%), 33 had a Matts grade of $3(26.6 \%), 27$ had a Matts grade of $4(21.8 \%)$, and 28 had a Matts grade of $5(22.6 \%)$. Titers of PR3-ANCA at Matts grade 1, 2, 3, 4, and 5 were 0 (0-3.4), 1.1 (0-3.2), 5.5 (0-15.1), 3.1 (0.7-7.0), and 7.4 (4.6-15.5), respectively. A positive correlation was observed between Matts grade and serum PR3-ANCA levels $(r=0.25 ; 95 \%$ confidence interval [Cl], 0.067-0.42; $p=0.0082)$.

Of 11 patients with cavitating ulcers, all patients had an MES of 3 , but 4 had a Matts grade of 3 , one had a Matts grade of 4 , and 6 had a Matts grade of 5. In this group, the titer of PR3-ANCA was 23.3 (2.2226.0), and 2 patients were negative for PR3-ANCA.

Of the 58 patients with active-phase PR3-ANCA-positive cases, 28 (48.3\%) were observed for 7.5 months (range, 5.1-15.0) and underwent endoscopic examination and PR3-ANCA measurements. Clinical remission was achieved in 21 of the 28 cases (75.0\%); however, 13 patients had an MES $\geq 1$ and only 8 patients had an MES of 0 (Table 5). 
Table 5

MES and PR3-ANCA positivity in clinical remission cases

\begin{tabular}{|lllll|}
\hline MES & $\mathbf{0}(\mathbf{n = 8})$ & $\mathbf{1}(\mathbf{n = 3})$ & $\mathbf{2}(\mathbf{n = 8})$ & $\mathbf{3}(\mathbf{n = 2})$ \\
\hline PR3-ANCA positive & 0 & 0 & 7 & 2 \\
\hline PR3-ANCA negative & 8 & 3 & 1 & 0 \\
\hline Abbreviation: MES, Mayo Endoscopic Subscore; \\
\hline
\end{tabular}

For MES 0 cases, the reduction of PR3-ANCA levels was significant $(p=0.012)$ when comparing the active-phase and clinical remission. In contrast, for MES $\geq 1$ cases, the reduction of PR3-ANCA levels was not significant ( $p=0.050$ ) (shown in Fig. 3). Of the 8 cases with MES 0 , all were negative for PR3-ANCA; only 4 of the 13 cases with MES $\geq 1$ (30.8\%) were negative for PR3-ANCA (Table 4). An endoscopic evaluation of activity of the 21 cases that reached clinical remission in PR3-ANCA-positive active-phase UC showed that PR3-ANCA had a sensitivity of $69.2 \%$, a specificity of $100 \%$, positive predictive value of $100 \%$, negative predictive value of $66.7 \%$, and a proper diagnosis rate of $81.0 \%$.

\section{Discussion}

The rate of positivity for PR3-ANCA in UC has been reported as $39.2 \%$ among Japanese individuals by Takedatsu et al[10]. In addition, it is reportedly $8.6 \%$ among Chinese individuals, $12.1 \%$ among Swedish individuals[20], and $14.1 \%$ among Spanish individuals[21]. In the present study considering Japanese individuals, the PR3-ANCA positivity rate was $53.7 \%$, which is close to that reported by Takedatsu et al. and higher than that reported in other countries.

Measurements of PR3-ANCA have been known to yield different results, dependent on the method used. Methods of measuring ANCA can be broadly classified as indirect immunofluorescence (IIF) and enzyme immunoassay (EIA). EIA methods include enzyme-linked immunosorbent assays (ELISA) and chemiluminescent enzyme immunoassays (CLEIA). IIF is reportedly unreliable because of changes in antigenicity or admixture of other antigens[22, 23]. Regarding EIA, CLEIA is reportedly more sensitive than ELISA for examinations of the same samples[24]. Although PR3-ANCA was measured using the same CLEIA method in the present study and by Takedatsu et al[10], other reports have not described the methods of measurement in detail. The differences in the positivity rates may be the result of differences in the measurement method.

Factors that affect the PR3-ANCA positivity rate in UC include patient background and UC activity. Important patient background characteristics are ethnicity, other diseases, and drugs used.

The onset of ANCA-associated vasculitis is often caused by silica exposure[25]. A relationship with chronic respiratory disease or malignant tumors has also been reported[18, 26]. Previous investigations of the relationship between UC and PR3-ANCA have not considered factors other than UC that could be 
PR3-ANCA-positive. The present study excluded cases involving complications that could affect PR3ANCA and cases involving drug use.

Drugs that require special attention with respect to ANCA include the following: hydralazine, an antihypertensive; propylthiouracil, a drug used to treat hyperthyroidism; minocycline, an antimicrobial; and TNF-a inhibitors $[27,28]$. TNF-a inhibitors are biologics that are used to treat UC and have an important relationship with ANCA. There have been reports of ANCA-associated vasculitis caused by infliximab or etanercept used to treat rheumatoid arthritis and juvenile idiopathic arthritis. Of note, TNF-a inhibitor use was an exclusion criterion in the present study. One patient with a non-active-phase case and a history of treatment with TNF-a inhibitors (excluded from the present study) had high PR3-ANCA levels $(58.6 \mathrm{U} / \mathrm{mL}$; data not shown).

The relationship between UC activity and PR3-ANCA remains controversial[10, 20]. As stated previously, results of previous reports may have been impacted by differences in the methods used to measure PR3ANCA, complications and the use of drugs. Although Takedatsu et al[10]. reported that PR3-ANCA is related to UC activity, UC activity was assessed according to clinical activity. In the present study, 21 cases entered clinical remission from the PR3-ANCA-positive active-phase of UC and had PR3-ANCA that was more reflective of endoscopic activity than clinical activity. The present study eliminated as many factors as possible that could affect the results. The UC group, which was PR3-ANCA-positive in the active-phase, had PR3-ANCA that was reflective of UC disease activity. However, $46.3 \%$ of PR3-ANCAnegative cases still had highly active UC; it is not known whether the heterogeneity of UC affects PR3ANCA production.

Colonoscopy evaluation is the gold standard for the assessment of disease activity of UC. A positive correlation was observed between MES and serum PR3-ANCA levels in this study. In addition, a positive correlation was observed between Matts grade and serum PR3-ANCA levels, although the correlation was weaker than that of MES. Some cases with a cavitating ulcer were underestimated when using the Matts grade. Matts grade is a pathological evaluation of the inflammation of the mucosa. Therefore, accurate assessment is difficult when evaluating the severe condition in which the ulcer reaches the muscular layer.

No prior study has investigated how PR3-ANCA affects inflammation of the mucosa caused by UC. Hyperactivity of neutrophils due to the formation and release of neutrophil extracellular traps (NETs) has been discussed as it pertains to the pathology of PR3-ANCA-associated vasculitis[6, 29, 30]. NETs are a network-like structure inside neutrophils with a mixture of neutrophil intranuclear DNA, neutrophil cytoplasmic MPO and PR3, neutrophil elastase, bactericidal/permeability-increasing protein, lactoferrin, and other antimicrobial proteins. In response to stimuli such as bacterial infection, NETs kill microbes by being released outside the cell in exchange for cell death[31]. However, NETs cause direct injury to vascular endothelial cells. Therefore, they are strictly controlled by being broken down, mainly by deoxyribonuclease I[32]. 
PR3-specific mouse monoclonal antibodies have been experimentally shown to assist with the formation of NETs[30]. Overproduction of NETs has been inferred to lower immune tolerance to MPO and PR3, consequently causing ANCA overproduction, which induces neutrophil hyperactivity and promotes inflammation[30, 33-35].

Although the underlying cause of UC remains unknown, large quantities of neutrophils are observed at the inflamed sites of the intestinal mucosa. In PR3-ANCA-positive active-phase UC in the present study, remission significantly decreased with PR3-ANCA levels, which was reflective of mucosal inflammation. In the UC group that became positive for PR3-ANCA during the active-phase, PR3-ANCA was expected to be involved in some inflammatory processes. It is inferred that PR3-ANCA exacerbates inflammation of the mucosa caused by UC, further complicating the mechanism of local inflammation of the mucosa; however, that mechanism remains unclear. In all cases in the present study, endoscopic images showed classic UC findings and biopsy results showed no findings of vasculitis.

PR3-ANCA-positive UC has been reported to involve few cases that are refractory or severe enough to require surgery or intervention with immunoregulators or biologics[36]. However, there have been reports of refractory cases and those that involve primary sclerosing cholangitis as a complication[24], but the relationship between the UC prognosis and PR3-ANCA remains controversial. The results of the present study showed that those who failed steroid therapy had a significantly higher proportion of PR3-ANCA positive individuals. ANCA-associated vasculitis is treated with combination therapy comprising steroids and immunoregulators[37, 38]. With PR3-ANCA-positive UC, it is possible that PR3-ANCA intensifies inflammation of the mucosa caused by UC, thus rendering steroid monotherapy insufficient to treat the active-phase. If this is the case, then PR3-ANCA in active-phase UC may be useful as an activity marker and could provide treating physicians with treatment ideas other than steroid monotherapy, such as biologics.

This study has several limitations. It is a retrospective observational study with short follow-up period and small sample size. We examined the significance of PR3-ANCA in relation to the response to the past therapy given against the most severe flare-up of colitis, but differences in the time of sampling may have affected the results. Furthermore, a single method was used to measure serum PR3-ANCA. Other methods of measurement need to be used to investigate whether the results are similar.

\section{Conclusions}

Approximately half of active-phase UC cases become positive for serum PR3-ANCA, thereby making it a potential marker for disease activity that may predict steroid therapy failure. Prospective studies must be performed to clarify the relationship between efficacy and PR3-ANCA for different methods of treating acute UC.

\section{List Of Abbreviations}


Alb albumin

ANCA anti-neutrophil cytoplasmic antibody

BMI body mass index

$\mathrm{Cl}$ confidence interval

CLEIA chemiluminescent enzyme immunoassays

CMV cytomegalovirus

CRP C-reactive protein

ElA enzyme immunoassay

ELISA enzyme-linked immunosorbent assays

IIF indirect immunofluorescence

LCAP leukocytapheresis

MES Mayo Endoscopic Subscore

MPO myeloperoxidase

MPO-ANCA myeloperoxidase anti-neutrophil cytoplasmic antibody

NETs neutrophil extracellular traps

Plt platelet

PR3 anti-proteinase 3

PR3-ANCA anti-proteinase 3 anti-neutrophil cytoplasmic antibody

ROC Receiver-operating characteristic

TNF tumor necrosis factor

UC ulcerative colitis

5-ASA 5-Aminosalicylic Acid;

\section{Declarations}


This study was approved by Ethics Committee of Kagawa Prefectural Central Hospital (No. 861) and Ethics Committee of Mitoyo General Hospital (No. 19CR01-101) and conducted in accordance with the Declaration of Helsinki. Written consent was obtained from all study participants.

\section{Consent for publication}

We obtained the consent for publication from all participants.

\section{Availability of data and materials}

The data sets generated and/or analyzed during the current study are available from the corresponding author on reasonable request.

\section{Competing interests}

The authors have no conflicts of interest to declare.

\section{Funding}

None.

\section{Authors' contributions}

YA, TI, and ST designed this study and wrote the manuscript. YA, TI, ST, HY, KI, SI and MW performed colonoscopy and assessed endoscopic findings. YA collected the data. TI supervised this study. ST is corresponding author. MA, SN and KM performed pathological examinations. $\mathrm{SH}$ and $\mathrm{HO}$ revised the manuscript for important intellectual content.

All authors have read and approved the final version to be published.

\section{Acknowledgments}

We would like to thank Editage (www.editage.com) for English language editing.

\section{References}

1) Mowat C, Cole A, Windsor A, Ahmad T, Arnott I, Driscoll R, et al. Guidelines for the management of inflammatory bowel disease in adults. Gut. 2011; 60: 571-607.

2) Frøslie KF, Jahnsen J, Moum BA, Vatn MH; IBSEN Group. Mucosal healing in inflammatory bowel disease : results from a Norwegian population-based cohort. Gastroenterology. 2007; 133: 412-22.

3) Nakarai A, Kato J, Hiraoka S, Kuriyama M, Akita M, Hirakawa T, et al. Evaluation of mucosal healing of ulcerative colitis by a quantitative fecal immunochemical test. Am J Gastroenterol. 2013; 108: 83-9. 
4) Takashima S, Kato J, Hiraoka S, Nakarai A, Takei D, Inokuchi T, et al. Evaluation of mucosal healing in ulcerative colitis by fecal calprotectin vs. fecal immunochemical Test. Am J Gastroenterol. 2015; 110: 873-80.

5) Lüdemann J, Utecht B, Gross WL. Detection and quantitation of anti-neutrophil cytoplasm antibodies in Wegener's granulomatosis by ELISA using affinity-purified antigen. J Immunol Methods. 1998; 114: 167-74.

6) Ishizu A. The pathogenesis of ANCA-associated vasculitis. J Clin Immunol. 2016; 39: 491-6.

7) Quinton JF, Sendid B, Reumaux D, Duthilleul P, Cortot A, Grandbastien B, et al. Anti-Saccharomyces cerecisiae mannan antibodies combined with antineutrophil cytoplasmic autoantibodies in inflammatory bowel disease: prevalence and diagnostic role. Gut. 1998; 42: 788-91.

8) Rump JA, Schölmerich J, Gross V, Roth M, Helfesrieder R, Rautmann A, et al. A new type of perinuclear anti-neutrophil cytoplasmic antibody (p-ANCA) in active ulcerative colitis but not in Crohn's disease. Immunobiology. 1990; 181: 406-13.

9) Xu J, Yang CH, Chen XY, Li XH, Dai M, Xiao SD. A subset of ulcerative colitis with positive proteinase-3 antineutrophil cytoplasmic antibody. World J Gastroenterol. 2008; 14: 7012-5.

10) Takedatsu H, Mitsuyama K, Fukunaga S, Yoshioka S, Yamauchi R, Mori A, et al. Diagnostic and clinical role of serum proteinase 3 antineutrophil cytoplasmic antibodies in inflammatory bowel disease. J Gastroenterol Hepatol. 2018; 33: 1603-7.

11) Mandl LA, Solomon DH, Smith EL, Lew RA, Katz JN, Shmerling RH. Using antineutrophil cytoplasmic antibody testing to diagnose vasculitis : can test-ordering guidelines improve diagnostic accuracy? Arch Intern Med. 2002; 162: 1509-14.

12) Shirai T, Takahashi R, Tajima Y, Kohata K, Yamamoto J, Fujii H, et al. Peripheral T cell lymphoma with a high titer of proteinase-3-antineutrophil cytoplasmic antibodies that resembled Wegener's granulomatosis. Intern Med. 2009; 48: 2041-5.

13) Pérez-De-Lis M, Retamozo S, Flores-Chávez A, Kostov $B$, Perez-Alvarez R, Brito-Zerón P, et al. Autoimmune diseases induced by biological agents. A review of 12,731 cases (BIOGEAS Registry). Expert Opin Drug Saf. 2017; 16: 1255-71.

14) Lennard-Jones JE. Classification of inflammatory bowel disease. Scand J Gastroenterol. 1989; 170: 2-6.

15) Satsangi J, Silverberg MS, Vermeire S, Colombel JF. The Montreal classification of inflammatory bowel disease: controversies, consensus, and implications. Gut. 2006;55:749-53. 
16) Schroeder KW, Tremaine WJ, Ilstrup DM. Coated oral 5-aminosalicylic acid therapy for mildly to moderately active ulcerative colitis. A randomized study. N Engl J Med. 1987; 317: 1625-9.

17) Matts SG. The value of rectal biopsy in the diagnosis of ulcerative colitis. Q J Med. 1961; 30: 393407.

18) Truelove SC, Jewell DP. Intensive intravenous regimen for severe attacks of ulcerative colitis. Lancet 1974; 1: 1067-70.

19) Munkholm P, Langholz E, Davidsen $M$, Binder V. Frequency of glucocorticoid resistance and dependency in Crohn's disease. Gut 1994; 35: 360-2.

20) Elzouki AN, Eriksson S, Löfberg R, Nässberger L, Wieslander J, Lindgren S. The prevalence and clinical significance of alpha 1-antitrypsin deficiency (PiZ) and ANCA specificities (proteinase 3, BPI) in patients with ulcerative colitis. Inflamm Bowel Dis. 1999; 5: 246-52.

21) García-Herola A, Nos P, Hoyos M, Hinojosa J, Molés JR, Pascual S, et al. Significance of the determination of antineutrophil cytoplasmic antibodies (ANCA) in ulcerative colitis and Crohn's disease. Gastroenterol Hepatol. 1998; 21: 169-73.

22) Cambridge G, Rampton DS, Stevens TR, McCarthy DA, Kamm M, Leaker B. Anti-neutrophil antibodies in inflammatory bowel disease: prevalence and diagnostic role. Gut. 1992; 33: 668-74.

23) Arimura Y, Marumo T, Fujii A, Nakabayashi K, Nagasawa T. Anti-neutrophil cytoplasmic antibody-enzyme immunosorbent assay. Rinsho Byori. 2001; 49: 571-4】

24) Stinton LM, Bentow C, Mahler M, Norman GL, Eksteen B, Mason AL, et al. PR3-ANCA : a promising biomarker in primary sclerosing cholangitis (PSC). PLoS One. 2014; 9: e112877. doi 10.1371/journal.pone.0112877.

25) Chen M, Kallenberg CG. The environment, geoepidemiology and ANCA-associated vasculitides. Autoimmun Rev. 2010; 9: A293-8.

26) Nada AK, Torres VE, Ryu JH, Lie JT, Holley KE. Pulmonary fibrosis as an unusual clinical manifestation of a pulmonary-renal vasculitis in elderly patients. Mayo Clin Proc. 1990; 65: 847-56.

27) Pendergraft WF 3rd, Herlitz LC, Thornley-Brown D, Rosner M, Niles JL. Nephrotoxic effects of common and emerging drugs of abuse. Clin J Am Soc Nephrol. 2014; 9: 1996-2005.

28) Hogan JJ, Markowitz GS, Radhakrishnan J. Drug-induced glomerular disease: immune-mediated injury. Clin J Am Soc Nephrol. 2015; 10: 1300-10.

29) Kessenbrock K, Krumbholz M, Schönermarck U, Back W, Gross WL, Werb Z, et al. Netting neutrophils in autoimmune small-vessel vasculitis. Nat Med. 2009; 15: 623-5. 
30) Nakazawa D, Shida H, Tomaru U, Yoshida M, Nishio S, Atsumi T, et al. Enhanced formation and disordered regulation of NETs in myeloperoxidase-ANCA-associated microscopic polyangiitis. J Am Soc Nephrol. 2014; 25: 990-7.

31) Brinkmann V, Reichard U, Goosmann C, Fauler B, Uhlemann Y, Weiss DS, et al. Neutrophil extracellular traps kill bacteria. Science. 2004; 303: 1532-5.

32) Hakkim A, Fürnrohr BG, Amann K, Laube B, Abed UA, Brinkmann V, et al. Impairment of neutrophil extracellular trap degradation is associated with lupus nephritis. Proc Natl Acad Sci USA. 2010; 107: 9813-8.

33) Döring Y, Weber C, Soehnlein O. Footprints of neutrophil extracellular traps as predictors of cardiovascular risk. Arterioscler Thromb Vasc Biol. 2013; 33: 1735-6.

34) Grayson PC, Kaplan MJ. At the Bench : Neutrophil extracellular traps(NETs) highlight novel aspects of innate immune system involvement in autoimmune disease. J Leukoc Biol. 2016; 99: 253-64.

35) Nakazawa D, Tomaru U, Suzuki A, Masuda S, Hasegawa R, Kobayashi T, et al. Abnormal conformation and impaired degradation of propylthiouracil-induced neutrophil extracellular traps: implications of disordered neutrophil extracellular traps in a rat model of myeloperoxidase antineutrophil cytoplasmic antibody-associated vasculitis. Arthritis Rheum. 2012; 64: 3779-87.

36) Arias-Loste MT, Bonilla G, Moraleja I, Mahler M, Mieses MA, Castro B, et al. Presence of antiproteinase 3 antineutrophil cytoplasmic antibodies(anti-PR3 ANCA) as serologic markers in inflammatory bowel disease. Clin Rev Allergy Immunol. 2013; 45: 109-16.

37) Mukhtyar C, Guillevin L, Cid MC, Dasgupta B, de Groot K, Gross W, et al. EULAR recommendations for the management of primary small and medium vessel vasculitis. Ann Rheum Dis. 2009; 68: 310-7.

38) Lapraik C, Watts R, Bacon P, Carruthers D, Chakravarty K, D'Cruz D, et al. BSR and BHPR guidelines for the management of adults with ANCA associated vasculitis. Rheumatology (Oxford). 2007; 46: 1615-6.

\section{Figures}




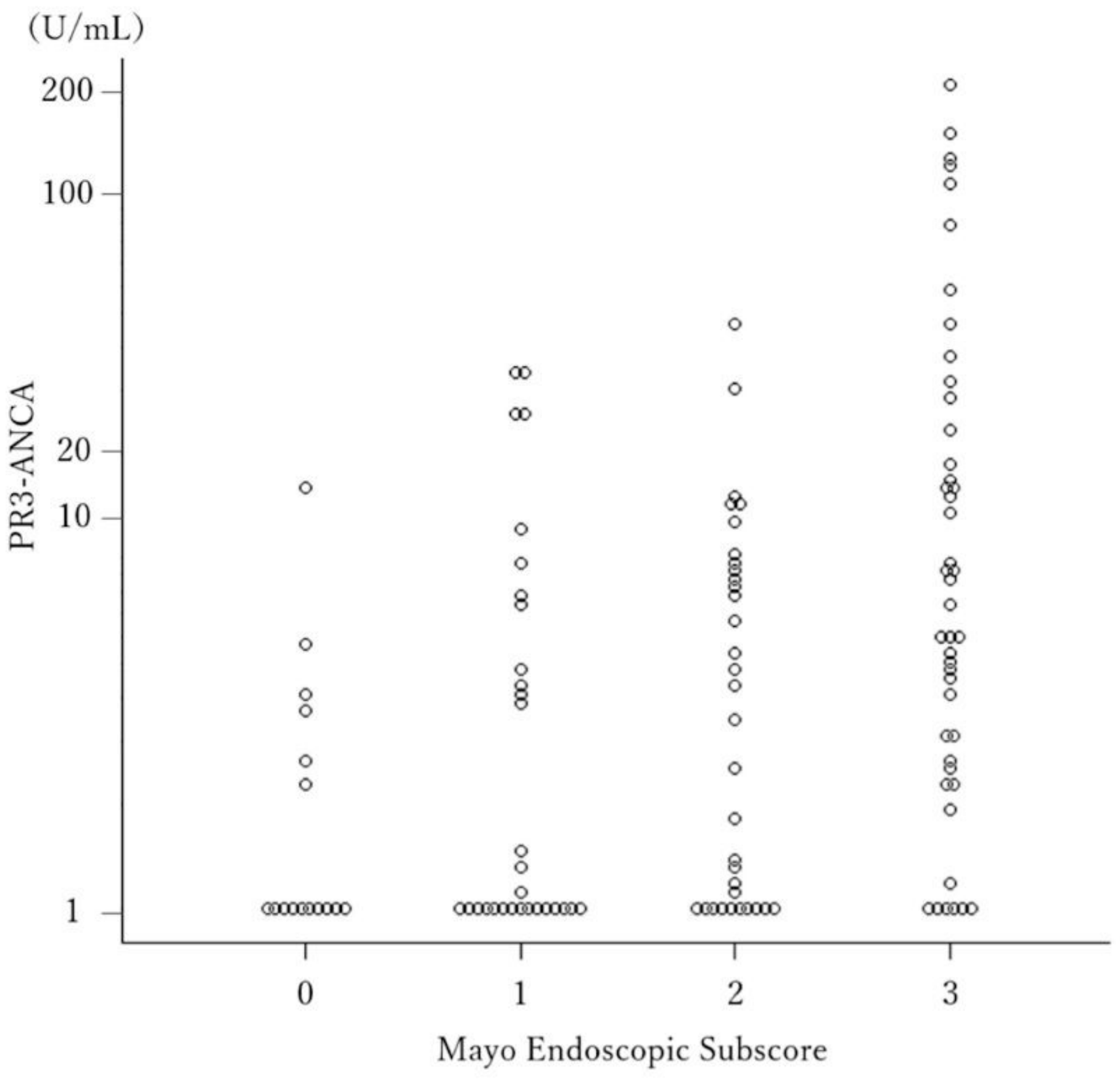

Figure 1

A positive correlation was observed between MES and serum PR3-ANCA levels $(r=0.42 ; 95 \% \mathrm{Cl}, 0.27-0.56$; $p<0.001)$. 


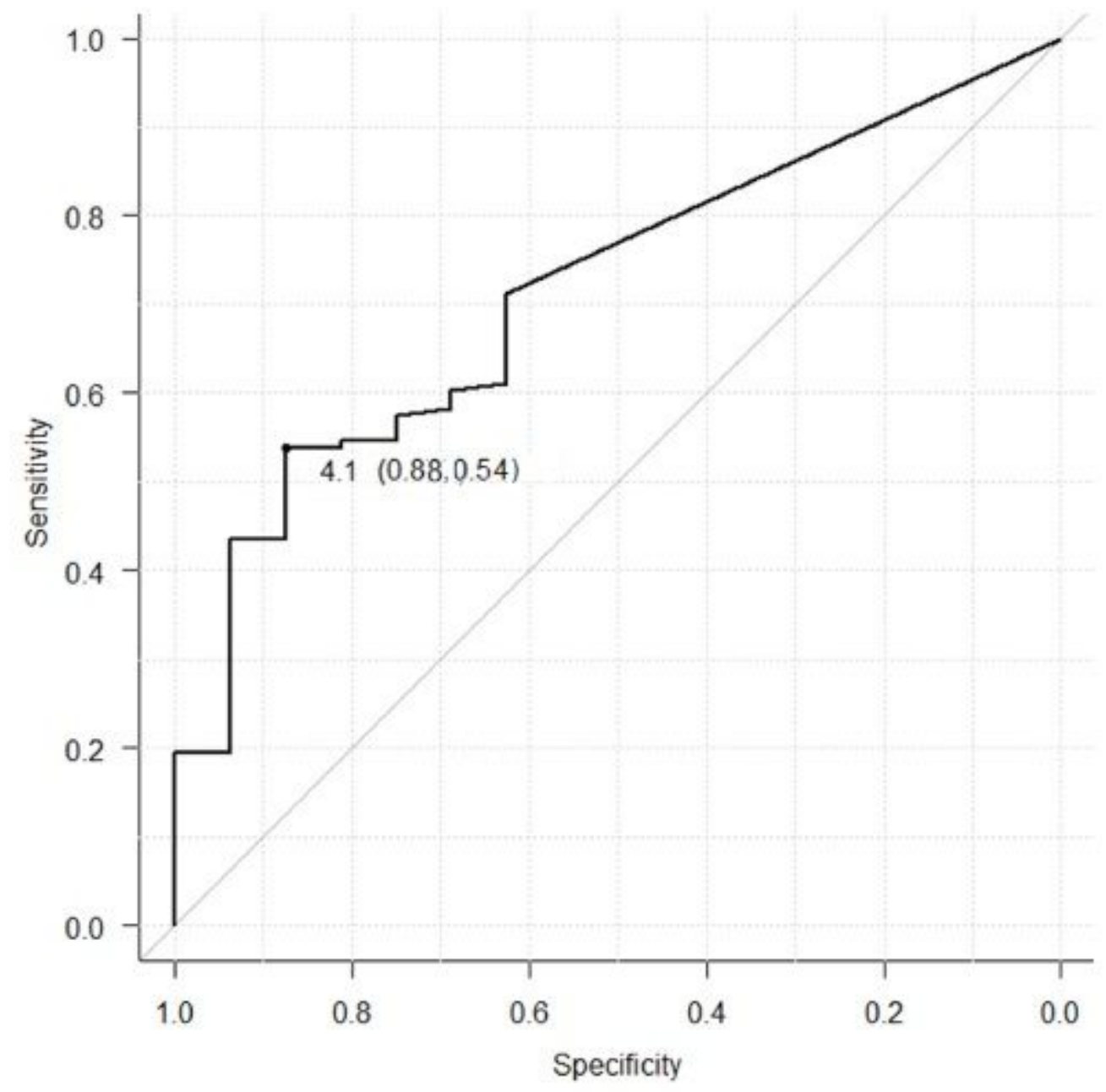

Figure 2

The ROC analysis indicated that the cut-off value was $4.1 \mathrm{U} / \mathrm{mL}$ (AUC, $0.72 ; 95 \% \mathrm{Cl}, 0.60-0.83$; sensitivity, 0.88 ; specificity, 0.54). 


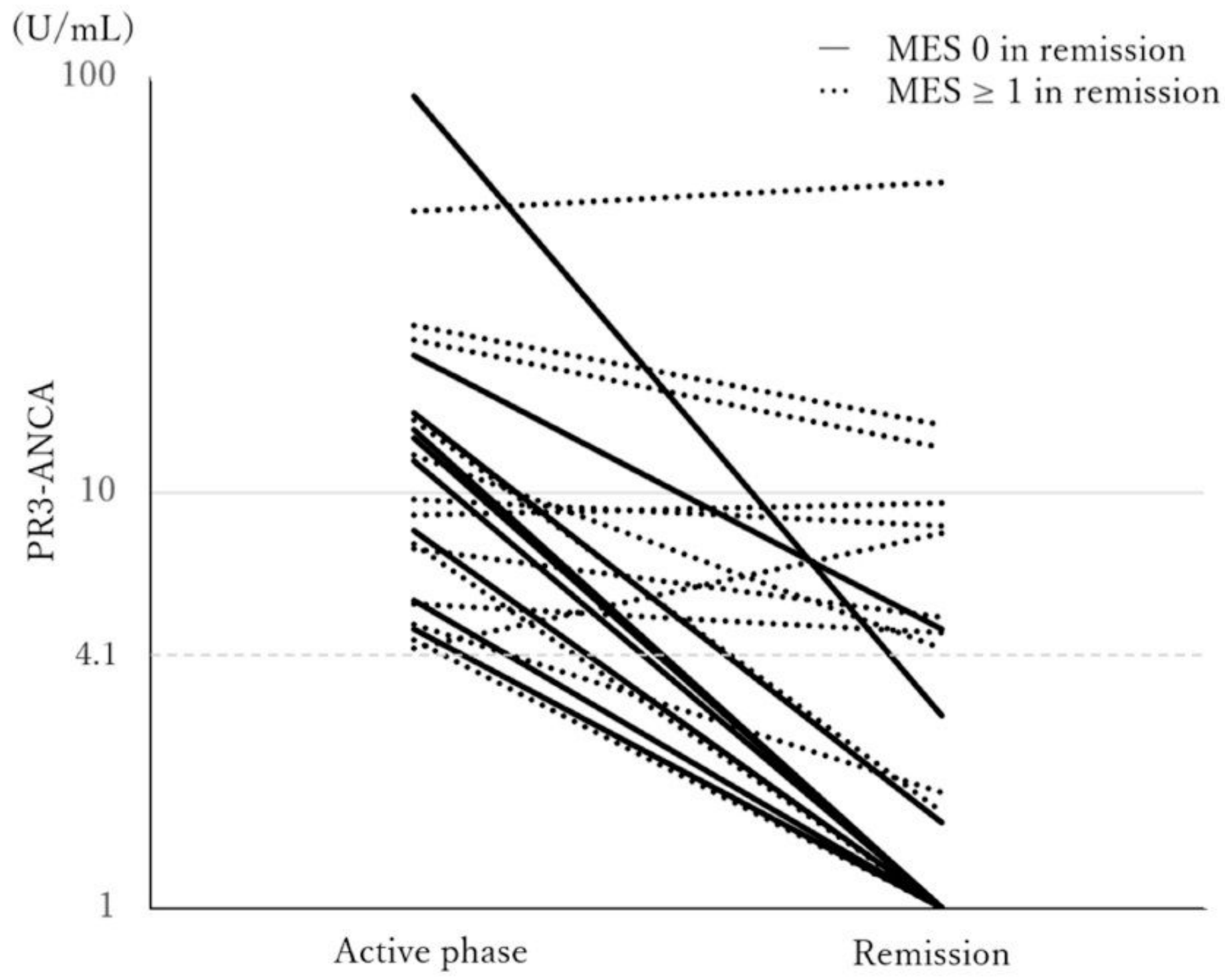

Figure 3

Thirteen cases had an MES $\geq 1$ and eight cases had an MES of 0 in remission. PR3-ANCA titers significantly declined $(p=0.012)$ and all became PR3-ANCA-negative in MES 0 cases. 